\title{
The boundary absolute continuity of quasiconformal mappings II
}

Juha Heinonen

Dedicated to Fred Gehring on his seventieth birthday

\begin{abstract}
In this paper a quite complete picture is given of the absolute continuity on the boundary of a quasiconformal map $\mathbb{B}^{3} \rightarrow D$, where $\mathbb{B}^{3}$ is the unit 3-ball and $D$ is a Jordan domain in $\mathbb{R}^{3}$ with boundary 2-rectifiable in the sense of geometric measure theory. Moreover, examples are constructed, for each $n \geq 3$, showing that quasiconformal maps from the unit $n$-ball onto Jordan domains with boundary $(n-1)$ rectifiable need not have absolutely continuous boundary values.
\end{abstract}

\section{Introduction.}

Suppose that $f$ is a quasiconformal homeomorphism of the open unit ball $\mathbb{B}^{n}$ of $\mathbb{R}^{n}$ onto a bounded domain $D$ in $\mathbb{R}^{n}$. Then $f$ extends homeomorphically to the boundary $\partial \mathbb{B}^{n}$ if and only if $D$ is bounded by a topological $(n-1)$-sphere [V1, p. 61]. Should such an extension exist, we denote it by $f$ as well, and call $D$ a Jordan domain or a quasiconformal Jordan ball. Suppose now that the boundary of $D$ has finite Hausdorff $\mathcal{H}_{n-1}$-measure. We say that $f$ is absolutely continuous on the boundary if $f$ carries sets of $\mathcal{H}_{n-1}$-measure zero on $\partial \mathbb{B}^{n}$ to sets of $\mathcal{H}_{n-1}$-measure zero on $\partial D$. If $n=2$ and $f$ is conformal, 
the boundary correspondence is absolutely continuous according to the classical theorem of $\mathrm{F}$. and M. Riesz [R]; but if $f$ is merely quasiconformal, it is well known, and first observed by Beurling and Ahlfors [BA], that the boundary correspondence need not be absolutely continuous even when $f$ is a self-homeomorphism of a disk. The situation is quite different in higher dimensions. For instance, if $f$ is a quasiconformal self-homeomorphism of $\mathbb{B}^{n}$, the boundary map is a quasiconformal map of $\mathbb{S}^{n-1}=\partial \mathbb{B}^{n}$ onto itself, and hence preserves sets of $(n-1)$-measure zero, provided $n-1 \geq 2$. It is therefore natural to ask what conditions on $\partial D$ are needed in order to have the absolute continuity of the boundary map $f: \partial \mathbb{B}^{n} \rightarrow \partial D$ when $n \geq 3$. For instance, is it sufficient that $\partial D$ be of finite $\mathcal{H}_{n-1}$-measure? In the present paper, which is a sequel to $[\mathrm{H}]$, a rather complete solution to this problem will be provided in dimension $n=3$ in the case when the boundary of $D$ is 2-rectifiable in the sense of geometric measure theory. It will also be shown that a direct analog of the F. and M. Riesz theorem is false for quasiconformal mappings in all dimensions. For the record, we shall only be dealing with the absolute continuity of the map $f: \partial \mathbb{B}^{n} \rightarrow \partial D$. It still remains widely open under what conditions the map $f^{-1}: \partial D \rightarrow \partial \mathbb{B}^{n}$ is absolutely continuous. Further open problems are listed in the end of the paper in Section 6.

Before proceeding, let us review the prior results in this area. So assume that $f$ is a quasiconformal mapping of $\mathbb{B}^{n}$ onto a Jordan domain $D$ whose boundary has finite $\mathcal{H}_{n-1}$-measure, and assume that $n \geq 3$. Gehring showed in [G2] that the boundary correspondence $f: \partial \mathbb{B}^{n} \rightarrow \partial D$ is absolutely continuous if $f$ has a quasiconformal extension to a neighborhood of $\partial \mathbb{B}^{n}$. Väisälä [V2] arrived at the same positive conclusion under the less restrictive assumption that $f$ be quasisymmetric on $\overline{\mathbb{B}}^{n}$. (Recall that quasisymmetry is a global condition as opposed to quasiconformality which is local; see (3.13) below for the definition of quasisymmetry.) In $[\mathrm{H}]$ it was shown that the answer is likewise affirmative if $\mathcal{H}_{n-1}$-almost every point on $\partial D$ is a "two sided cone point", and if $n \neq 4$. To make this supposition more precise, we next fix some notation. Let $L$ be a line in $\mathbb{R}^{n}$ through a point $a$ and let $0<s<1$. Set

$$
\mathcal{C}(a, L, s)=\left\{x \in \mathbb{R}^{n}: \operatorname{dist}(x, L)<s|a-x|\right\} .
$$

The point $a$ divides the line $L$ into two pieces, which we shall call $L^{+}$ and $L^{-}$. The orientation of the line plays no role in our arguments, so this choice is arbitrary. We write

$$
\mathcal{C}\left(a, L^{+}, s\right)=\left\{x \in \mathbb{R}^{n}: \operatorname{dist}\left(x, L^{+}\right)<s|a-x|\right\},
$$


and similarly for $\mathcal{C}\left(a, L^{-}, s\right)$. Thus $\mathcal{C}(a, L, s)$ is the union of the two infinite open cones $\mathcal{C}\left(a, L^{+}, s\right)$ and $\mathcal{C}\left(a, L^{-}, s\right)$ with $s$ determining the angle opening. We also use the notation

$$
\begin{aligned}
\mathcal{C}(a, r, L, s) & =\mathcal{C}(a, L, s) \cap B(a, r), \\
\mathcal{C}\left(a, r, L^{ \pm}, s\right) & =\mathcal{C}\left(a, L^{ \pm}, s\right) \cap B(a, r) .
\end{aligned}
$$

Here and throughout $B(z, t)$ will denote the open $n$-ball which is centered at $z$ and has radius $t>0$.

We say that a set $E \subset \mathbb{R}^{n}$ has a double cone at a point $a \in E$, or that $a$ is a double cone point of $E$, if there are $L, s$, and $r$, possibly depending on $a$, such that $E \cap \mathcal{C}(a, r, L, s)=\varnothing$.

The following theorem was proved in $[\mathrm{H}]$.

Theorem 1.1. Suppose that $n=3,5,6, \ldots$ and that $f$ is a quasiconformal mapping of $\mathbb{B}^{n}$ onto a Jordan domain $D$. Let $\mathcal{C}_{D}$ denote the set of double cone points of $\partial D$. Then for any set $A \subset \mathcal{C}_{D}$ we have that $\mathcal{H}_{n-1}(A)=0$ if and only if $\mathcal{H}_{n-1}\left(f^{-1}(A)\right)=0$. In particular, if $\mathcal{H}_{n-1}$-almost every point of $\partial D$ is a double cone point of $\partial D$, then the boundary map $f: \partial \mathbb{B}^{n} \rightarrow \partial D$ is absolutely continuous.

It follows from Theorem 1.1 in particular that if $\partial D$ admits a tangent plane at $\mathcal{H}_{n-1}$ almost every point, then the boundary correspondence of $f$ is absolutely continuous. If $f$ is quasisymmetric, it follows from the results in [V2] that $\partial D$ admits tangents almost everywhere, if it has finite $\mathcal{H}_{n-1}$ measure. Hence Theorem 1.1 contains the aforementioned results of Gehring and Väisälä in dimensions $n \neq 4$. The proof in [H] works in all dimensions $n \geq 3$ for mappings that are bi-Lipschitz in the quasihyperbolic metric; by the aid of the Sullivan-Tukia-Väisälä approximation theorem the general quasiconformal case can be reduced to this case in dimensions different from four. Unfortunately, I have not been able to dispense with this reduction, and consequently, there is no proof of Theorem 1.1 in dimension $n=4$ (see Added in Proof at the end of the paper).

Geometric measure theory has taught us that the right concept of rectifiability is expressed in terms of "approximate tangents". If $E \subset \mathbb{R}^{n}$, we say that an $(n-1)$-plane $V$ in $\mathbb{R}^{n}$ is an approximate tangent plane for $E$ at $a$ if $a$ is a point of $\mathcal{H}_{n-1}$ density of $E$ and if

$$
\lim _{r \rightarrow 0} \frac{\mathcal{H}_{n-1}(E \cap B(a, r) \backslash \mathcal{C}(a, V, s))}{r^{n-1}}=0,
$$


for all $0<s<1$, where

$$
\mathcal{C}(a, V, s)=\left\{x \in \mathbb{R}^{n}: \operatorname{dist}(x, V)<s|a-x|\right\}
$$

If such a plane $V$ exists, it is unique and we denote it by $\operatorname{apTan}(E, a)$. Intuitively, $\operatorname{ap} \operatorname{Tan}(E, a)$ is a plane that approximates $E$ near $a$ except for some leftover part which has zero $\mathcal{H}_{n-1}$-density along each cone with vertex at $a$ and axis perpendicular to the plane apTan $(E, a)$.

In this paper we shall call a set $(n-1)$-rectifiable (or sometimes simply rectifiable if there is no danger of misunderstanding the dimension of the set) if it has finite $\mathcal{H}_{n-1}$-measure and if it admits an approximate tangent plane at $\mathcal{H}_{n-1}$-almost all of its points. We refer to $[\mathrm{F}]$ and $[\mathrm{M}]$ for more information about rectifiable sets. (Warning: the terminology in both $[\mathrm{F}]$ and $[\mathrm{M}]$ is slightly different.) It suffices to mention here that a set $E$ of finite $\mathcal{H}_{n-1}$-measure in $\mathbb{R}^{n}$ is $(n-1)$-rectifiable if and only if it is contained in a countable union of Lipschitz images of $\mathbb{R}^{n-1}$ inside $\mathbb{R}^{n}$. Moreover, every set of finite $\mathcal{H}_{n-1}$-measure can be decomposed into a rectifiable and a purely unrectifiable part, the latter being a set whose intersection with any rectifiable set in $\mathbb{R}^{n}$ has zero $\mathcal{H}_{n-1}$-measure.

Next, we say that a boundary point $a$ of a domain $D$ is an inner cone point if there are $L, s$ and $r$ such that $\mathcal{C}\left(a, r, L^{+}, s\right)$ lies in $D$. Moreover, we say that $a$ is an inner tangent point of $D$ if there is a line $L$ with the following property: for each $s<1$ there is $r>0$ such that $\mathcal{C}\left(a, r, L^{+}, s\right)$ lies in $D$. In this case the half line $L^{+}$can be called an interior normal line to $\partial D$ at $a$. Naturally, $L^{+}$need not be unique.

The following theorem is the first main result of this paper.

Theorem 1.2. Suppose that $f$ is a quasiconformal mapping of $\mathbb{B}^{3}$ onto a Jordan domain $D$ with 2-rectifiable boundary. Then we have a decomposition of $\partial D$ into three disjoint sets,

$$
\partial D=E_{0} \cup E_{1} \cup E_{2},
$$

where $E_{0}$ has $\mathcal{H}_{2}$-measure zero, $E_{1}$ consists of points of inner tangency of $D$, and $E_{2}$ consists of points of 3-density of $\mathbb{R}^{3} \backslash D$. The Hausdorff dimension of $f^{-1}\left(E_{2}\right)$ is zero, and for a set $A \subset E_{1}$ the preimage $f^{-1}(A)$ has $\mathcal{H}_{2}$-measure zero if and only if $A$ has $\mathcal{H}_{2}$-measure zero.

In other words, if $D$ is a Jordan domain in $\mathbb{R}^{3}$ with 2-rectifiable boundary and if $f$ maps $\mathbb{B}^{3}$ quasiconformally onto $D$, then, apart from an $\mathcal{H}_{2}$-null set, the boundary $\partial D$ consists of the "good part", where $f$ 
and $f^{-1}$ both are absolutely continuous, and the "bad part", which is easily detected and which is responsible for the possible failure of the absolute continuity of $\left.f\right|_{\partial \mathbb{B}^{3}}$. Thus the only way the absolute continuity can fail for domains with rectifiable boundary is to have a situation where the bad part $E_{2}$ is non-empty and has positive $\mathcal{H}_{2}$-measure. The next theorem says that such situations can occur.

Theorem 1.3. For each $n \geq 3$ there is a Jordan domain $D$ in $\mathbb{R}^{n}$ such that $D$ is quasiconformally equivalent to $\mathbb{B}^{n}$, that $\partial D$ is $(n-1)$ rectifiable, and the set

$$
E_{2}=\left\{a \in \partial D: a \text { is a point of } n \text {-density of } \mathbb{R}^{n} \backslash D\right\}
$$

has positive $\mathcal{H}_{n-1}$-measure. Moreover, the preimage $f^{-1}\left(E_{2}\right)$ under any quasiconformal map ff from $\mathbb{B}^{n}$ onto $D$ has Hausdorff dimension zero.

Theorem 1.3 answers negatively to an inquiry of Baernstein and Manfredi [BM, p. 846]. It also shows that Theorem 1.1 is quite sharp. (Note that if $\partial D$ has finite $\mathcal{H}_{n-1}$-measure and if it admits double cones at $\mathcal{H}_{n-1}$-almost everywhere, then it is $(n-1)$-rectifiable; see [M, Lemma 15.13]). Of course, it is easy to construct Jordan domains with the measure theoretic properties as in Theorem 1.3; the nontrivial part is to show that some of them can be mapped quasiconformally onto a ball.

The fact that $f^{-1}\left(E_{2}\right)$ has Hausdorff dimension zero in Theorems 1.2 and 1.3 is a recent result of Koskela and Rohde [KR]. They prove, among other things, that the preimage of the set $E_{2}$ as described in (1.4) has zero Hausdorff dimension always; that is, in all dimensions and for all quasiconformal mappings $f: \mathbb{B}^{n} \rightarrow D$ (with boundary values properly interpreted if $D$ is not Jordan). In our situation, it would be much easier to show that $f^{-1}\left(E_{2}\right)$ has $\mathcal{H}_{n-1}$-measure zero. In fact, the method described in this paper shows that one can construct a domain $D$ as in Theorem 1.3 such that $\mathcal{H}_{n-1}\left(E_{2}\right)$ is positive and that $f^{-1}\left(E_{2}\right)$ has zero Hausdorff $\mathcal{H}_{h}$-measure for any prescribed Hausdorff measure function $h$. The construction of the domain is based on the ideas of Väisälä in [V4], where he constructed a quasiconformal Jordan ball whose boundary has positive $n$-measure. The elaboration of Väisälä's method presented here leads to a general "tree and pipeline" procedure to build quasiconformal balls and may be of independent interest.

One may ask whether the assumption in Theorem 1.2 that $\partial D$ be 2 -rectifiable can be relaxed to the assumption that $\mathcal{H}_{2}(\partial D)$ be finite. I do not know the answer. An example can be constructed to show that 
the assumptions in Theorem 1.2 cannot be relaxed to " $D$ is Jordan and $\partial D$ has $\sigma$-finite Hausdorff $\mathcal{H}_{n-1}$-measure".

I conjecture that Theorem 1.2 is true in all dimensions $n \geq 3$. In the present paper, the argument for Theorem 1.2 relies in a crucial way on the following local description of the boundary of a quasiconformal Jordan ball (see Added in Proof at the end of the paper).

Theorem 1.5. Suppose that $D$ is a Jordan domain in $\mathbb{R}^{3}$ which is homeomorphic to $\mathbb{B}^{3}$ via a $K$-quasiconformal map. Then for each $x \in D$ we have the estimate

$$
\mathcal{H}_{2}(B(x, 2 \operatorname{dist}(x, \partial D)) \cap \partial D) \geq C(K) \operatorname{dist}(x, \partial D)^{2} .
$$

Theorem 1.5 is interesting in its own right. It quantifies the fact that the boundary of a quasiconformal ball cannot have lower dimensional parts protruding inwards. It has also led Jussi Väisälä to make general conjectures about isodiametric inequalities for sets that satisfy certain connectivity conditions; see (6.1) below. I make the following conjecture involving quasiconformal mappings.

1.7. Wall Conjecture for Quasiconformal Balls. If $D$ is a domain in $\mathbb{R}^{n}$ that is homeomorphic to $\mathbb{B}^{n}$ via a $K$-quasiconformal map, then for each $x \in D$ we have the estimate

$$
\mathcal{H}_{n-1}(B(x, 2 \operatorname{dist}(x, \partial D)) \cap \partial D) \geq C(n, K) \operatorname{dist}(x, \partial D)^{n-1} .
$$

Note that the conjecture is true for $n=2$ for quite trivial reasons; namely, there is a big connected piece of the boundary inside $B(x, 2 \operatorname{dist}(x, \partial D))$. The conjecture is also true for $n=1$, when properly interpreted. Despite some effort, I have not been able to prove the conjecture for $n \geq 4$. Assuming that it is true even in the weaker form where the constant $C(n, K)$ in (1.8) is allowed to depend on $D$, the proof for Theorem 1.2 will work mutatis mutandis for all $n \geq 5$. Dimension $n=4$ has to be excluded for the same reason it is excluded in $[\mathrm{H}]$ : at some point in the proof we need to resort to the fact that in dimensions $n \neq 4$ quasiconformal maps of $\mathbb{B}^{n}$, say, can be replaced by locally bi-Lipschitz quasiconformal maps without changing the boundary values. 
It follows from Theorem 1.5 and standard capacity estimates that every quasiconformal Jordan ball in $\mathbb{R}^{3}$ is regular for the Dirichlet problem for the Laplacian; in fact, it is regular for the $p$-Laplace equation for all $p>1$. It is not true that an arbitrary Jordan domain in $\mathbb{R}^{3}$ is regular for the Laplacian as the well known Lebesgue's spine demonstrates. If the Wall Conjecture 1.7 is true, then quasiconformal Jordan balls are regular for the $p$-Laplacian in all dimensions and for all $p>1$. I thank Pekka Koskela for pointing out this application.

Finally, I wish to point out the recent interesting paper by Hanson [Ha], where rectifiability (Hanson uses a weaker notion here) of the boundary of a quasiconformal Jordan ball is tied up with the behavior of the average derivative $a_{f}$ in the classical spirit. Recall that the rectifiability of a Jordan curve $\Gamma$ in the plane is equivalent to the membership of $f^{\prime}$ in the Hardy class $H^{1}$ for any conformal map $f$ from the unit disk onto the domain bounded by $\Gamma$. Hanson proves in $[\mathrm{Ha}]$ that among all quasiconformal Jordan balls $D$ in $\mathbb{R}^{n}, n \geq 3$, that are also so-called uniform domains, the finiteness of $\mathcal{H}_{n-1}(\partial D)$ is equivalent to the membership of the average derivative $a_{f}$ in a "Hardy space" $H^{n-1}$, if $f$ maps $\mathbb{B}^{n}$ quasiconformally onto $D$. (We refer to [Ha] for a precise definition for these concepts.) Many relations between $a_{f}$, rectifiability and absolute continuity remain to be sorted out. In particular, it is plausible that Hanson's theorem indeed requires some extra assumptions on $D$. Hanson [Ha, (5.8) p. 140-141] also advances a conjecture about quasiconformal mappings that is similar to the wall conjecture 1.7. It is not clear what the relationship between these two conjectures are.

\section{Outline of Proof for Theorem 1.2.}

In this section the main points in the proof for Theorem 1.2 are sketched for the expert's convenience.

Assume that the Wall Conjecture 1.7 is true. We know that $\mathcal{H}_{n-1^{-}}$ almost every point on $\partial D$ has an approximate tangent plane. We let $E_{0}$ be the exceptional set, and $E_{2}$ the set consisting of the points of $n$-density of the complement of $D$. For the first part of the theorem, it suffices to show that each point in $E_{1}=\partial D \backslash E_{0} \cup E_{2}$ is a point of inner tangency for $D$. If this is not the case, there is a point $a \in E_{1}$ and a line $L$ which is perpendicular to $\operatorname{ap} \operatorname{Tan}(\partial D, a)$ and so oriented that the cone $\mathcal{C}\left(a, r, L^{+}, s\right)$ intersects the boundary $\partial D$ for arbitrarily 
small $r>0$ and for some fixed $s$. By (1.8), to each Whitney cube of the open set $D_{r}=D \cap \mathcal{C}\left(a, r, L^{+}, s\right)$ there corresponds a substantial piece of the boundary lying in a cone $\mathcal{C}\left(a, r, L^{+}, s^{\prime}\right)$ with somewhat bigger opening $s^{\prime}<1$. Because $a$ is not a point of density of $\mathbb{R}^{n} \backslash D$, the set $D_{r}$ is indeed nonempty and has $n$-measure comparable to $r^{n}$. Now the boundary pieces are essentially disjoint, and their $\mathcal{H}_{n-1}$-measures add up to something which is comparable to $r^{n-1}$. This contradicts the fact that $\partial D$ admits an approximate tangent plane at $a$.

The second assertion of Theorem 1.2 is an improvement to Theorem 1.1 which says that $f$ and $f^{-1}$ preserve sets of zero $\mathcal{H}_{n-1}$-measure on double cone points. The proof given in $[\mathrm{H}]$ requires double cone points, but, below in Section 3, I give a sharpening of that argument which only needs interior cones, in the presence of approximate tangents. The technical argument of $[\mathrm{H}]$ can be shortened somewhat, but the basic idea is still the same. Suppose, for instance, that there is a subset $A$ of interior cone points of $\partial D$ of positive $\mathcal{H}_{n-1}$-measure such that $f^{-1}(A)$ has zero $\mathcal{H}_{n-1}$-measure. After a standard reduction, we may assume that $A$ lies on the boundary of a bi-Lipschitz ball contained in $D$, hence we may assume without loss of generality that $A$ lies on the boundary of a round ball $B$ contained in $D$. Then we use the assumption that $n \neq 4$ and replace $f$ by a locally bi-Lipschitz quasiconformal homeomorphism $F$ which agree with $f$ on the boundary. The technical point, as in $[\mathrm{H}]$, is to show that $F^{-1}(B)=\Omega$ is a uniform domain with "nice" boundary in $\mathbb{B}^{n}$; the niceness is defined in terms of the following Ahlfors-David regularity condition:

$$
C^{-1} R^{n-1} \leq \mathcal{H}_{n-1}(B(x, R) \cap \partial \Omega) \leq C R^{n-1}
$$

for each $x \in \partial \Omega$ and $0<R<\operatorname{diam} \Omega$. This condition and known results on quasisymmetric maps onto regular surfaces guarantee that $\left.F\right|_{\partial \Omega}$ is absolutely continuous, contradicting the hypothesis. In establishing this technical point, we use a Hayman-Wu type "spotting" technique and a Carleson measure argument; the main difference from $[\mathrm{H}]$ is that now we have to make use of the approximate tangent planes in place of the exterior cones. More details will follow in the next section.

\section{Proof of Theorem 1.2.}

The ensuing proof works in all dimensions $n \geq 3$, under right assumptions. Thus, assume that $f$ is a quasiconformal mapping from 
$\mathbb{B}^{n}$ onto a Jordan domain $D$ with rectifiable boundary. Also assume that $D$ satisfies (1.8) for some constant $C$, possibly depending on $D$. By Theorem 1.5 this is always true in dimension $n=3$. Then the conclusion is that the boundary $\partial D$ decomposes as in Theorem 1.2 with $f^{-1}\left(E_{2}\right)$ having Hausdorff dimension zero. If in addition $n \neq 4$, then the absolute continuity of $\left.f\right|_{f^{-1}\left(E_{1}\right)}$ and $\left.f^{-1}\right|_{E_{1}}$ is also true as in Theorem 1.2.

To begin the proof, let $E_{0}$ denote the set on $\partial D$ where $\partial D$ does not admit approximate tangent planes. Then $E_{0}$ has $\mathcal{H}_{n-1}$-measure zero. We divide $\partial D \backslash E_{0}$ into two subsets $E_{1}$ and $E_{2}$, where $E_{2}$ consists of the points of $n$-density of the complement of $D$ in $\mathbb{R}^{n}$, and $E_{1}$ is what remains. Our first task will be to show that every point in $E_{1}$ is a point of inner tangency for $D$.

\subsection{Inner tangency of points in $E_{1}$.}

Pick a point $a \in E_{1}$. Let $L$ be the line through $a$ which is perpendicular to the approximate tangent plane for $\partial D$ at $a$. Fix $0<s<1$. We need to show that there is $r>0$ such that one of the two components of the double cone $\mathcal{C}(a, r, L, s)$ is contained in $D$. Because $a$ is not a point of $n$-density for the complement of $D$, and because $\partial D$ has finite $\mathcal{H}_{n-1}$-measure, we can assume, by making $s$ larger if necessary and by choosing an appropriate orientation for $L$, that

$$
\limsup _{r \rightarrow 0} \frac{\mathcal{H}_{n}\left(\mathcal{C}\left(a, r, L^{+}, s\right) \cap D\right)}{r^{n}}>0 .
$$

Next, we suppose that

$$
\partial D \cap \mathcal{C}\left(a, r, L^{+}, s\right) \neq \varnothing
$$

for all $r>0$ and then show that this leads to a contradiction with the fact that

$$
\lim _{r \rightarrow 0} \frac{\mathcal{H}_{n-1}\left(\partial D \cap \mathcal{C}\left(a, r, L^{+}, s^{\prime}\right)\right)}{r^{n-1}}=0
$$

for all $0<s^{\prime}<1$.

To this end, let $D_{r}=\mathcal{C}\left(a, r, L^{+}, s\right) \cap D$ and observe that $D_{r} \neq \varnothing$ by (3.2). Suppose first that for each $x \in D_{r}$ the ball $B_{x}=B(x, \operatorname{dist}(x, \partial D))$ satisfies

$$
\operatorname{diam} B_{x} \leq \varepsilon \operatorname{dist}\left(B_{x}, a\right)
$$


where $\varepsilon=\varepsilon(s)>0$ is so small that (3.4) implies

$$
2 B_{x} \subset \mathcal{C}\left(a, 2 r, L^{+},(1+s) / 2\right) .
$$

By standard covering theorems (see [M, Chapter 2]), we can choose a countable collection $\left\{B_{i}: i=1,2, \ldots\right\}$ of balls of the form $B_{x}$ such that

$$
D_{r} \subset \bigcup_{i} 2 B_{i}
$$

and that

$$
\sum_{i} \chi_{2 B_{i}}(x) \leq C(n) .
$$

The latter condition simply says that no point in $\mathbb{R}^{n}$ belongs to more than $C(n)$ balls of the form $2 B_{i}$. Therefore, by assumption (1.8),

$$
\begin{aligned}
\mathcal{H}_{n-1}\left(\partial D \cap \mathcal{C}\left(a, 2 r, L^{+},(1+s) / 2\right)\right) & \geq C^{-1} \sum_{i} \mathcal{H}_{n-1}\left(\partial D \cap 2 B_{i}\right) \\
& \geq C^{-1} \sum_{i}\left(\operatorname{diam} B_{i}\right)^{n-1} \\
& \geq C^{-1}\left(\sum_{i}\left(\operatorname{diam} B_{i}\right)^{n}\right)^{(n-1) / n} \\
& \geq C^{-1}\left(\mathcal{H}_{n}\left(D_{r}\right)\right)^{(n-1) / n} .
\end{aligned}
$$

Because the constant $C \geq 1$ above is independent of $r>0$, we contradict (3.3) with the aid of (3.2).

We may thus assume that

$$
\operatorname{diam} B_{x}>\varepsilon \operatorname{dist}\left(B_{x}, a\right)
$$

for some $x \in D_{r}$ and $B_{x}=B(x, \operatorname{dist}(x, \partial D))$. In this case a simple geometric argument proves the existence of a point $y \in D_{r}$ and a ball $B_{y}=B(y, \operatorname{dist}(y, \partial D))$ that belongs to $\mathcal{C}\left(a, r, L^{+}, s\right)$ and satisfies both,

$$
2 B_{y} \subset \mathcal{C}\left(a, 2 r, L^{+},(1+s) / 2\right)
$$

and

$$
C^{-1} \operatorname{diam} B_{y} \leq \operatorname{dist}\left(B_{y}, a\right) \leq C \operatorname{diam} B_{y},
$$

for some $C \geq 1$ depending only on $n$ and $s$. Thus we deduce that, for some $r^{\prime}<r$,

$$
\mathcal{H}_{n-1}\left(\partial D \cap \mathcal{C}\left(a, 2 r^{\prime}, L^{+},(1+s) / 2\right)\right) \geq \mathcal{H}_{n-1}\left(\partial D \cap 2 B_{y}\right) \geq C^{-1} r^{\prime n-1}
$$


by assumption (1.8). Moreover, $C \geq 1$ is independent of $r$ and $r^{\prime}$. This again contradicts (3.3) and we have shown that $a$ is a point of inner tangency of $D$.

\subsection{Absolute continuity in the inner tangency set $E_{1}$.}

Recall that the fact that $f^{-1}\left(E_{2}\right)$ has Hausdorff dimension zero is due to Koskela and Rohde [KR]. To complete the proof of the theorem, it thus remains to show that $f$ and $f^{-1}$ are absolutely continuous in the sets $f^{-1}\left(E_{1}\right) \subset \partial \mathbb{B}^{n}$ and $E_{1} \subset \partial D$. The proof here has the same idea as in $[\mathrm{H}]$. In that paper, however, the absolute continuity was proved in the set of double cone points, and the existence of an exterior cone was also essentially used there. In the present situation we only have an interior cone to rely on. The supporting role of the exterior cone is taken here by the approximate tangent plane, which exists at each point in $E_{1}$. This change forces us to make some technical modifications to the proof in $[\mathrm{H}]$. It would be unreasonable to repeat here all the details of $[\mathrm{H}]$, and I apologetically ask the reader to consult that paper whenever necessary. The good news is that the most technical part of the proof of [H, Lemma 3.1] has now been simplified somewhat.

Let us begin with the following lemma.

Lemma 3.6. Let a be a point in $E_{1}$ and denote by $T_{a}$ the approximate tangent plane apTan $(\partial D, a)$. Then

$$
\limsup _{r \rightarrow 0} \inf _{v \in T_{a},|v-a|=r} \frac{\operatorname{dist}(v, \partial D)}{|v-a|}=0 .
$$

Proof. This lemma looks trivial but a little thinking shows that it need not be true if we replace $\partial D$ by an arbitrary $(n-1)$-rectifiable set. In any event, the ensuing proof is quite easy.

Suppose on the contrary that there is $\delta>0$ and a sequence of radii $\left(r_{i}\right), r_{i} \rightarrow 0$ as $i \rightarrow \infty$, such that

$$
\frac{\operatorname{dist}(v, \partial D)}{|v-a|}>\delta
$$

for all $v \in T_{a}$ with $|v-a|=r_{i}$. Then the $\delta r_{i}$-neighborhood $U_{i}$ of the $(n-2)$-sphere $\partial B\left(a, r_{i}\right) \cap T_{a}$ does not meet $\partial D$. Because $a$ is a point of inner tangency of $D$, it follows that $U_{i} \subset D$ for all large $i$. Let $L^{+}$be the interior normal line at $a$, perpendicular to $T_{a}$. Fix $s$ so close to 1 that 
$U_{i}$ meets $\mathcal{C}\left(a, L^{+}, s\right)$ for all large $i$, and then choose $r_{i}>0$ so that the cone $\mathcal{C}\left(a, r_{i}, L^{+}, s\right)$ is contained in $D$. Clearly $\mathcal{C}\left(a, r_{i}, L^{-}, s\right)$ cannot be contained in $D$, for otherwise the connected open set $U_{i} \cup \mathcal{C}\left(a, r_{i}, L, s\right)$ is contained in $D$ for all large $i$ and separates the point $a$ from the part of the boundary that lies outside $B\left(a, r_{i}\right)$. Thus, for arbitrary small $r_{i}>0$ we have that

$$
\mathcal{C}\left(a, r_{i}, L^{-}, s\right) \cap \partial D \neq \varnothing
$$

Using assumption (1.8) on the thickness of the boundary, this leads to a contradiction with an argument similar to that in the end of the proof in (3.1). (Note: the analog of (3.1) in the present case is guaranteed by the size of $U_{i}$.) The lemma follows.

\subsection{Reduction to a ball.}

Suppose now that $A \subset E_{1}$ has positive $\mathcal{H}_{n-1}$ measure. We need to show that $f^{-1}(A)$ has positive $\mathcal{H}_{n-1}$-measure as well. And this is in fact all that needs to be shown in detail, for the case

$$
A \subset E_{1} \text { and } \mathcal{H}_{n-1}(A)=0 \text { implies } \quad \mathcal{H}_{n-1}\left(f^{-1}(A)\right)=0
$$

is treated similarly.

A standard measure theoretic trick guarantees that there is a subset $A_{0} \subset A$ of positive $\mathcal{H}_{n-1}$-measure which lies on the boundary of a bounded starshaped subdomain $\Omega_{0} \subset D$. The domain $\Omega_{0}$ can be mapped onto a ball by a bi-Lipschitz self-map of $\mathbb{R}^{n}$. Because biLipschitz maps preserve rectifiability and sets of positive Hausdorff measure, we can assume, originally, that $A$ lies on the boundary of a ball $B_{0}$ contained in $D$. See [H, Proof of Theorem 4.3] for more details here.

Next we form a Stolz domain $\Omega$ in $B_{0}$, associated with $A$ the usual way. That is, $\Omega$ consists of all the open rays with one end point in $B_{0} / 2$ and the other in $A$. Then $\Omega$ is a bi-Lipschitz ball contained in $D$ and containing $A$ on its boundary. (Note that the round ball $B_{0}$ already satisfies these conditions and it would be nice if we could manage with $B_{0}$ alone. It is the proof below in (3.14) that needs a domain like $\Omega$ which is safely inside $B_{0}$.) 


\subsection{Bi-Lipschitz maps in the quasihyperbolic metric.}

Now we use the assumption $n \neq 4$. The Sullivan-Tukia-Väisälä approximation theorem [TV2, 7.12] provides us with a quasiconformal map $F: \mathbb{B}^{n} \rightarrow D$ such that

$$
k_{D}(f(x), F(x)) \leq 1
$$

and that

$$
C^{-1} k_{D}(F(x), F(y)) \leq k_{\mathbb{B}^{n}}(x, y) \leq C k_{D}(F(x), F(y))
$$

for all $x$ and $y$ in $\mathbb{B}^{n}$ and for some $C=C(n, f) \geq 1$. Here $k_{G}$ denotes the quasihyperbolic metric in a domain $G$, defined by the metric density $\operatorname{dist}(x, \partial G)^{-1}|d x|$.

Condition (3.9) guarantees that $f$ and $F$ have the same boundary values and (3.10) says that $F$ is bi-Lipschitz in the quasihyperbolic metrics. We deduce that there is no loss of generality in assuming, originally, that the mapping $f$ satisfies (3.10).

\subsection{Regular surfaces and subinvariance.}

Write $g=f^{-1}$. The main bulk of the proof consists of showing that the boundary $\partial g(\Omega)$ is an Ahlfors-David $(n-1)$-regular set; that is, there is a constant $C \geq 1$ such that

$$
C^{-1} R^{n-1} \leq \mathcal{H}_{n-1}(B(x, R) \cap \partial g(\Omega)) \leq C R^{n-1},
$$

for all $x \in \partial g(\Omega)$ and $0<R<\operatorname{diam} g(\Omega)$.

Suppose for a moment that this has been accomplished. The proof is then finished as follows. The subinvariance principle for quasiconformal maps guarantees that $\left.g\right|_{\Omega}: \Omega \rightarrow g(\Omega)$ is a quasisymmetric map, which means that

$$
|x-y| \leq t|x-z| \quad \text { implies }|g(x)-g(y)| \leq \eta(t)|g(x)-g(z)|
$$

for all points $x, y, z \in \Omega$ and for some homeomorphism $\eta:[0, \infty) \rightarrow$ $[0, \infty)$. (See [FHM, p. 120-121] and [V3, Theorem 5.6]). Clearly (3.13) will continue to hold for all points in the closure $\bar{\Omega}$, so that $\left.g\right|_{\partial \Omega}: \partial \Omega \rightarrow$ $\partial \Omega$ is quasisymmetric as well. Because $\partial \Omega$ is a bi-Lipschitz $(n-1)$ sphere and because $\partial g(\Omega)$ is $(n-1)$-regular in the sense of $(3.12)$, we can 
invoke known results about quasisymmetric maps in such situations to conclude that $\mathcal{H}_{n-1}(E)>0$ if and only if $\mathcal{H}_{n-1}(g(E))>0$ for $E \subset \partial \Omega$. (See $[\mathrm{S}, 3.4]$ or $[\mathrm{H}, 2.7]$ ).

We conclude, therefore, that it remains to prove the regularity (3.12) of $\partial g(\Omega)$.

\subsection{Proof of regularity of $\partial g(\Omega)$.}

We begin by making two more reductions. Fix $\varepsilon$ positive and small, to be determined later. By Lemma 3.6 there is, for each $a \in A$, a positive number $r_{a}$ such that

$$
\operatorname{dist}\left(v_{r}, \partial D\right)<\varepsilon\left|v_{r}-a\right|
$$

for some $v_{r} \in T_{a} \cap \partial B(a, r)$ and for all $0<r \leq r_{a}$. Then

$$
A=\bigcup_{j=1}^{\infty}\left\{a \in A: r_{a}>1 / j\right\}
$$

and we may assume that there is $\delta>0$ such that $r_{a}>\delta>0$ for all $a \in A$. We assume further that each point in $A$ is a point of $\mathcal{H}_{n-1^{-}}$ density on $\partial B_{0}$ (recall that $A$ lies in the smooth hypersurface $\partial B_{0}$ and has positive $\mathcal{H}_{n-1}$-measure).

The left inequality in (3.12) follows from the quasisymmetry of $g$ in $\partial \Omega$ by a result of Väisälä [V2, 5.2]. The right inequality in (3.12) follows by standard arguments using (3.10) from the following lemma (for the details, see [H, p. 1564-65]).

Lemma 3.16. Suppose that $\left(x_{i}\right)$ is a hyperbolically separated sequence of points on $\partial \Omega$; this means that there is $\tau>0$ such that

$$
B\left(x_{i}, \tau \operatorname{dist}\left(x_{i}, \partial D\right)\right) \cap B\left(x_{j}, \tau \operatorname{dist}\left(x_{j}, \partial D\right)\right)=\varnothing
$$

whenever $i \neq j$. Then there is $C \geq 1$ such that

$$
\sum_{g\left(x_{i}\right) \in B}\left(1-\left|g\left(x_{i}\right)\right|\right)^{n-1} \leq C(\operatorname{diam} B)^{n-1},
$$

for all $n$-balls $B$ centered on $\partial \mathbb{B}^{n}$. 
One should notice that in (3.18) the constant $C \geq 1$ can, and usually will, depend on everything else but on $B$.

Thus, fix a ball $B$, centered at some point on $\partial \mathbb{B}^{n}$. Henceforth $C$ will denote any positive constant that is independent of $B$ and also of index $i$. The goal is to find for each $x_{i}$ in $g^{-1}(B)=f\left(B \cap \mathbb{B}^{n}\right)$ its own spot $S_{i}$ on $\partial D$ such that the following three conditions hold:

$$
\sum_{i} \chi_{S_{i}}(x) \leq C
$$

that is, no point in $\mathbb{R}^{n}$ belongs to more that $C$ spots $S_{i}$;

$$
g\left(S_{i}\right) \subset C B \cap \partial \mathbb{B}^{n},
$$

that is, the image of each spot $S_{i}$ will not land far from $B$ under the map $g$; and

$$
\left(1-\left|g\left(x_{i}\right)\right|\right)^{n-1} \leq C \mathcal{H}_{n-1}\left(g\left(S_{i}\right)\right)
$$

that is, the Hausdorff measure of the image $g\left(S_{i}\right) \subset \partial \mathbb{B}^{n}$ essentially dominates the term $\left(1-\left|g\left(x_{i}\right)\right|\right)^{n-1}$ of the sum in (3.18).

It is clear that (3.18) follows from (3.19)-(3.21).

Before we start describing the spots $S_{i}$ with desired properties, we make two observations.

\subsection{Hyperbolic freedom.}

There is never any harm in replacing any of the points $x_{i}$ by a point $\tilde{x}_{i}$ for which

$$
k_{D}\left(x_{i}, \tilde{x}_{i}\right) \leq C,
$$

because it is easily seen that (3.23) implies

$$
\left(1-\left|g\left(x_{i}\right)\right|\right) \leq C\left(1-\left|g\left(\tilde{x}_{i}\right)\right|\right) .
$$

The replacement may cause us to diminish the value of $\tau$ in (3.17) a little bit, but such adjustments are left to the reader. 


\subsection{Generational gaps.}

Upon dividing $\left(x_{i}\right)$ into generations $\mathcal{G}_{\nu}$,

$x_{i} \in \mathcal{G}_{\nu} \quad$ if and only if $\quad \operatorname{dist}\left(x_{i}, \partial B_{0}\right) \in\left(2^{-\nu-1}, 2^{-\nu}\right], \quad \nu \in \mathbf{Z}$,

we can assume that

$$
\mathcal{G}_{\nu}=\varnothing \quad \text { for } \quad \nu \leq C,
$$

and that

$$
\mathcal{G}_{\nu_{i}} \neq \varnothing \neq \mathcal{G}_{\nu_{j}} \quad \text { implies } \quad \nu_{i}=\nu_{j} \text { or }\left|\nu_{i}-\nu_{j}\right| \geq C .
$$

Above, $C$ should be thought of as a large constant, to be adjusted later. Condition (3.26) means that we only have to worry about those points $x_{i}$ that lie near the boundary of $B_{0}$, and (3.27) says that we can assume that there are large generational gaps. In short, we assume that $\mathcal{G}_{\nu}$ is nonempty only if $\nu$ is positive and a constant multiple of a large integer. We shall construct the spots $S_{i}$ in such a way that $S_{i}$ and $S_{j}$ are disjoint whenever they correspond to points in different generations, and that the finite overlap condition (3.19) holds for spots $S_{i}$ corresponding to points from the same generation.

\subsection{Determining points $z_{i}$.}

We shall associate to each point $x_{i}$ in our sequence two more points, $w_{i}$ and $z_{i}$, of which the latter will play a more important role. To get a mental picture,

$$
x_{i} \quad \rightsquigarrow \quad w_{i} \in \partial B_{0} \quad \rightsquigarrow \quad z_{i} \in \partial D .
$$

The point $w_{i}$ is simply the closest point to $x_{i}$ on $\partial B_{0}$, and $z_{i}$ is a closest point to $w_{i}$ on $\partial D$. Of course, it may happen that $w_{i}=z_{i}$. Before we fix these, however, we need to make some adjustments to the sequence $\left(x_{i}\right)$ in the spirit of $(3.22)$.

Thus, pick a point $x_{i}$. Let $w_{i}$ be the closest point to $x_{i}$ on $\partial B_{0}$, and let $a_{i}$ be the closest point to $x_{i}$ on $A$. Because each point in $A$ is assumed to be a point of $\mathcal{H}_{n-1}$-density, it is clear that the approximate tangent plane $T_{a_{i}}$ is also tangent to $\partial B_{0}$ at $a_{i}$. By choosing the constant $C$ in (3.26) large enough, we may assume that

$$
\operatorname{dist}\left(w_{i}, T_{a_{i}}\right)<\varepsilon\left|w_{i}-a_{i}\right|,
$$


where $\varepsilon>0$ is as in (3.15).

Let $w_{i}^{\prime}$ be the point where the ray emanating from the center of $B_{0}$ and passing through $x_{i}$ meets $T_{a_{i}}$, and let

$$
r_{i}^{\prime}=\left|a_{i}-w_{i}^{\prime}\right|
$$

Again, by making the constant $C$ in (3.26) large enough, we may assume that $r_{i}^{\prime}<\delta$ for all $i$, where $\delta$ is defined just after (3.15). Thus we can find a point $v_{i} \in T_{a_{i}} \cap \partial B\left(a_{i}, r_{i}^{\prime}\right)$ such that

$$
\operatorname{dist}\left(v_{i}, \partial D\right)<\varepsilon\left|v_{i}-a_{i}\right|
$$

Now let $\tilde{x}_{i}$ be the point on the line segment from $v_{i}$ to the center of $B_{0}$ such that

$$
\operatorname{dist}\left(\tilde{x}_{i}, \partial B_{0}\right)=\operatorname{dist}\left(x_{i}, \partial B_{0}\right) .
$$

It is easy to see that

$$
k_{D}\left(x_{i}, \tilde{x}_{i}\right) \leq C .
$$

Therefore, by the discussion 3.22, we may assume, originally, that

$$
\operatorname{dist}\left(w_{i}^{\prime}, \partial D\right)<\varepsilon\left|w_{i}^{\prime}-a_{i}\right|
$$

and hence that

$$
\operatorname{dist}\left(w_{i}, \partial D\right) \leq\left|w_{i}-w_{i}^{\prime}\right|+\varepsilon\left|w_{i}^{\prime}-a_{i}\right| \leq 2 \varepsilon\left|w_{i}-a_{i}\right|
$$

provided that $C$ in (3.26) is large enough, depending on $\varepsilon$. Next, let $z_{i}$ be a point on $\partial D$ such that

$$
\left|z_{i}-w_{i}\right|=\operatorname{dist}\left(w_{i}, \partial D\right)
$$

and observe that

$$
\left|z_{i}-w_{i}\right| \leq 2 \varepsilon\left|w_{i}-a_{i}\right|
$$

At this point we could invoke the argument in [H, Main Lemma 3.1] which applies in the present situation. The double cone condition there was used only to guarantee the existence of the points $z_{i}$ satisfying (3.30). For the reader's convenience, however, I shall sketch below a somewhat different and perhaps easier argument for the rest of the proof. 
Towards this end, we require the following lemma which is proved in $[\mathrm{HK}, 6.6]$.

Lemma 3.31. Let $x \in D$. There is a constant $C \geq 1$, depending only on $n$ and on the dilatation of $f$, such that

$$
\mathcal{H}_{n-1}\left(g(B(x, C \operatorname{dist}(x, \partial D)) \cap \partial D) \cap \Delta_{g(x)}\right) \geq \frac{1}{2} \mathcal{H}_{n-1}\left(\Delta_{g(x)}\right),
$$

where $\Delta_{g(x)}$ is the surface cap,

$$
\Delta_{g(x)}=B(g(x), 3(1-|g(x)|)) \cap \partial \mathbb{B}^{n} .
$$

Now fix $0<\lambda<1$ and let $u_{i}$ be the point $(1-\lambda) z_{0}+\lambda w_{i}$ in $B_{0}$, where $z_{0}$ is the center of $B_{0}$. We have

$$
\operatorname{dist}\left(u_{i}, \partial D\right) \leq\left|u_{i}-w_{i}\right|+\left|w_{i}-z_{i}\right|=(1-\lambda)+2 \varepsilon\left|w_{i}-a_{i}\right| .
$$

By choosing $\lambda=\lambda_{i}$ such that

$$
\left(1-\lambda_{i}\right)=\varepsilon \operatorname{dist}\left(x_{i}, \partial B_{0}\right)
$$

we find that

$(3.32) \operatorname{dist}\left(u_{i}, \partial D\right) \leq \varepsilon \operatorname{dist}\left(x_{i}, \partial B_{0}\right)+2 \varepsilon\left|w_{i}-a_{i}\right| \leq 10 \varepsilon\left|w_{i}-a_{i}\right|$.

Finally, define $S_{i}$ by

$$
g\left(S_{i}\right)=g\left(B\left(u_{i}, C \operatorname{dist}\left(u_{i}, \partial D\right)\right) \cap \partial D\right) \cap \Delta_{g\left(u_{i}\right)},
$$

where $C$ is as in Lemma 3.31. We easily infer by choosing $\varepsilon>0$ small enough, by observing the generational gap (3.27), Lemma 3.31, formula (3.32), and the geometry of $\Omega$ that this choice of $S_{i}$ will satisfy (3.19)(3.21). Of course, we need to observe here that

$$
k_{D}\left(u_{i}, x_{i}\right) \leq C,
$$

as well as the assumed hyperbolic separation (3.17) of the points $x_{i}$. The details are left to the interested reader.

This completes the proof of Theorem 1.2. 


\section{A class of quasiconformal balls - proof of Theorem 1.3.}

In this section I exhibit a general method to build quasiconformal balls. As mentioned before, the method described below is essentially due to Väisälä who constructed a single interesting example in [V4]; the main idea of blowing up towers with moderate dilatation is of course old and goes back to the early articles of Gehring and Väisälä [GV]. My contribution is simply to axiomatize the construction done in [V4], and then point out how one obtains this way examples that are relevant to the boundary absolute continuity problem.

\subsection{Admissible trees.}

An admissible tree in $\mathbb{R}^{n}$ is a tree around which one can build a quasiconformal ball. A precise definition follows shortly. In the ensuing discussion, all line segments are assumed to be finite and closed. We shall work in $\mathbb{R}^{n}$ for any $n$ bigger than one, although the Riemann mapping theorem trivializes the discussion for $n=2$.

Let $L_{0}$ be a line segment in $\mathbb{R}^{n}$ and fix $\alpha \in(0, \pi / 2]$. Set $\mathcal{J}_{0}=$ $\left\{L_{0}\right\}$. Suppose next that a finite collection $\mathcal{J}_{i}$ of line segments has been determined for all $i=0, \ldots, k$. Let $L \in \mathcal{J}_{k}$ be a line segment. Attach a finite number of line segments $L_{1}, \ldots, L_{p}$ to $L$ in such a way that

1) exactly one of the end points of each $L_{i}$ lies on $L \backslash\{$ the end points of $L$;

2 ) the angle between each $L_{i}$ and $L$ is at least $\alpha>0$;

3) all line segments $L_{i}$ are mutually disjoint and none of them meets any line segment from $\mathcal{J}_{0} \cup \cdots \cup \mathcal{J}_{k}$ except their parent $L$ at one end point.

We further stipulate that all the children of all line segments from $\mathcal{J}_{k}$ as described above are mutually disjoint; they form the collection $\mathcal{J}_{k+1}$.

We call the set

$$
T_{\alpha}=T=\bigcup_{k=1}^{\infty} \mathcal{J}_{k}
$$

an admissible tree with branching angle $\alpha$ if it is a bounded set, constructed by the above rules 1)-3), and has the additional property that each line segment $L$ from $T$ retains a positive distance (depending on $L$ ) to all other line segments from $T$, save its immediate family (that 
is, its parent and children); more formally,

$$
\operatorname{dist}(L, T \backslash\{\text { the parent of } L \text { and the children of } L\})>0
$$

for each $L \in T$. We understand that $L_{0}$ has no parent and that there can be childless line segments.

Next, denote by $F_{T}$ the set of all points that lie "behind infinitely many branches". More precisely, $x$ is in $F_{T}$ if $x$ is a cluster point of infinitely many line segments from $T$.

Theorem 4.3. Given an admissible tree $T_{\alpha}=T$ in $\mathbb{R}^{n}$, there is a domain $D$ in $\mathbb{R}^{n}$ such that

$$
T \subset \bar{D}
$$

that

$$
F_{T} \subset \partial D
$$

and that $D$ is quasiconformally equivalent to $\mathbb{B}^{n}$ by a $K$-quasiconformal map with $K$ depending only on $n$ and $\alpha$.

Even more can be said.

Theorem 4.6. Given any admissible tree $T_{\alpha}=T$ in $\mathbb{R}^{n}$, any continuous nondecreasing function $h:[0,1) \rightarrow[0,1), h(t) \rightarrow 0$ as $t \rightarrow 0$, and any $\varepsilon>0$, there is a domain $D$ in $\mathbb{R}^{n}$ satisfying (4.5) and (4.6), and there is a quasiconformal map $f$ from $\mathbb{B}^{n}$ onto $D$ such that

$$
\mathcal{H}_{h}\left(f^{-1}\left(F_{T}\right)\right)=0
$$

and that

$$
\mathcal{H}_{n-1}\left(\partial D \backslash F_{T}\right)<\varepsilon \text {. }
$$

Moreover, one can choose $f$ such that its dilatation depends only on $n$ and $\alpha$.

Above, $\mathcal{H}_{h}$ denotes the Hausdorff measure obtained from the measure function $h$; see [F, 2.10].

Accepting Theorems 4.3 and 4.6, it is easy to construct examples as in Theorem 1.3. For instance, one can take a totally disconnected compact set $F$ in $\mathbb{R}^{n-1}$ with positive $\mathcal{H}_{n-1}$-measure, and then form an 
admissible tree having branches in the upper half space $\mathbb{R}_{+}^{n}=\left\{x \in \mathbb{R}^{n}\right.$ : $\left.x_{n}>0\right\}$ such that the end points of these branches capture each point in $F$. It follows from the construction below that the boundary of the associated domain $D$ is a rectifiable $(n-1)$-sphere, and that one can arrange each point on $F$ to be a point of $n$-density for the complement of $D$.

Many other interesting examples of quasiconformal balls can be exhibited by the aid of the above theorems. For instance, the existence of quasiconformal Jordan balls with boundary having positive $n$-measure is ascertained by the existence of pertinent admissible trees. Väisälä's goal in [V4] was exactly to construct one such domain. Väisälä was partly motivated by the following consequence of his construction: there are mappings in the Sobolev space $W_{l o c}^{1, n}\left(\mathbb{R}^{n} ; \mathbb{R}^{n}\right)$ that do not preserve sets of $n$-measure zero. Theorem 4.6 can be used to show that mappings in $W^{1, n}$ can blow up quite a miniscule set to a set of positive $n$-measure. This is done by "folding" a mapping promised in Theorem 4.6, cf. [Re], [V4, p. 206]. For a general discussion on this topic, see [MM].

We shall only prove Theorem 4.3. It should be clear how the details need to be changed in order to achieve Theorem 4.6. Overall, we shall rely on the carefully detailed argument in [V4].

\subsection{Tower maps.}

For $h>0$ define the straight tower

$$
T(h)=\Delta \cup\left(\overline{\mathbb{B}}^{n-1} \times[0, h]\right) \subset \mathbb{R}^{n},
$$

where $\Delta=\left(-e_{n}\right) \overline{\mathbb{B}}^{n-1}$ is the join of $-e_{n}=(0, \ldots, 0,-1)$ and the closed unit ball of $\mathbb{R}^{n-1}$. If $\alpha \in(0, \pi / 2]$, a leaning tower $T(h, \alpha)$ is obtained from the straight tower $T(h)$ by keeping the base $\Delta$ fixed and tilting the upper part $\overline{\mathbb{B}}^{n-1} \times[0, h]$ so that it makes angle $\alpha$ with the hyperplane $\mathbb{R}^{n-1}$. We call $\Delta$ the basement, and $\overline{\mathbb{B}}^{n-1}$ the floor, of the tower $T(h, \alpha)$. The terms wall and roof of $T(h, \alpha)$ are selfexplanatory when we make the convention that both these sets consist only of points where $\partial T(h, \alpha)$ is smooth, i.e. we ignore the corners.

A tower map is a quasiconformal map

$$
g: \Delta \rightarrow T(h, \alpha)
$$

such that $g$ is the identity on the part of $\partial \Delta$ that does not include $\overline{\mathbb{B}}^{n-1}$. Strictly speaking, $g$ is quasiconformal only in the interior of the 
basement $\Delta$, but it extends so as to map $\Delta$ homeomorphically onto $T(h, \alpha)$.

The existence of such a map is clear; what is crucial is that it can be chosen so that its dilatation only depends on $n$ and $\alpha_{0}$, if $\alpha \geq \alpha_{0}>0$. In particular - and this is the main point - the dilatation does not depend on the height $h$ of the (leaning) tower $T(h, \alpha)$. Moreover, we can choose $g$ such that it is a diffeomorphism at every point in the preimage of the wall of the tower. For an explicit construction of the map $g$, see [V4, Section 3].

\subsection{Flattening of walls and germs of similarity.}

Suppose that a leaning tower $T(h, \alpha)$ is given and that $\left\{a_{1}, \ldots, a_{p}\right\}$ is a finite subset of the wall of $T(h, \alpha)$. One can modify both the tower and the tower map in (4.10) so that it becomes a similarity in small neighborhoods of the points $a_{i}^{\prime}=g^{-1}\left(a_{i}\right) \in \overline{\mathbb{B}}^{n-1}$. This is done as follows. First one flattens out a small piece of the slightly curved wall surface near each point $a_{i}$. This does not cost much in terms of the dilatation. Then, using the language of Väisälä, one can plant a germ of similarity on $g$ near each point $a_{i}^{\prime}$. This means that one can modify the map $g$ so that it becomes a similarity (in particular, conformal) in a neighborhood of $a_{i}^{\prime}$. Moreover, the planting can be done in such a way that the cost in dilatation only depends on $n$ and the dilatation of the original map, that is, on $n$ and $\alpha$ only in our case.

In sum, we can assume that given a tower as above and a finite number of points on its wall, we have a tower map

$$
g: \Delta \rightarrow T^{\prime}(h, \alpha)
$$

where the new tower $T^{\prime}(h, \alpha)$ is being slightly flattened around the given points. (We could call $T^{\prime}(h, \alpha)$ a tilted pajupilli.) Moreover, $g$ is a similarity near those points and its dilatation only depends on $n$ and a lower bound for the tilt angle of the tower. On the part of the boundary of the basement that lies in the lower half space, the map $g$ is still the identity.

The planting procedure is being described in detail in [V4, Section $2]$. 


\subsection{Proof of Theorem 4.3.}

Once we have the tower map (4.12) at our disposal, it is rather clear how to continue the proof. Suppose that we are given an admissible tree $T_{\alpha}=T$. First we map the unit ball under a quasiconformal map $f_{0}$ onto a thin cylinder $\mathcal{C}_{0}$ about $T_{0}$ such that the height of the cylinder is the length of $T_{0}$ and that $T_{0}$ is its axis. The dilatation of $f_{0}$ only depends on $n$, and not on the height. We choose the cylinder $\mathcal{C}_{0}$ so thin that all the children of $L_{0}$ in $\mathcal{J}_{1}$ stick out of it a good proportion of their length, and that all the other descendants remain at a positive distance from $L_{0}$; this is possible by (4.2). We reiterate that $\mathcal{C}_{0}$ can be made as thin as we please with no extra cost at the dilatation of $f_{0}$. Consequently, the surface area of $\mathcal{C}_{0}$ can be made as small as we please; this observation is needed for Theorem 4.6.

Next, at the points $a_{i}$, where the children $L_{i}$ of $L_{0}$ leave the cylinder $\mathcal{C}_{0}$, we flatten the wall of $L_{0}$ and assume, as we may by the discussion in 4.11 , that $f_{0}^{-1}$ is a similarity in a neighborhood $U_{i}$ of each point $a_{i}$. We place small similarity copies $\Delta_{i}$ of $\Delta$ in all those neighborhoods $U_{i}$ such that the origin in $\Delta$ corresponds to $a_{i}$ in $\Delta_{i}$. Usually the child $L_{i}$ leaves the cylinder $\mathcal{C}_{0}$ in a tilt, and we place a thin leaning tower on each $\Delta_{i}$ such that $L_{i}$ is the axis of the tower and that the other end point of $L_{i}$ lies on the roof of the tower. Any such tower is a similarity copy of a tower of the form $T(h, \alpha)$ described above in 4.9. We choose these towers so thin that they do not meet other descendants but their immediate children; again this is possible by (4.2).

Each base $\Delta_{i}$ can be mapped quasiconformally onto the leaning tower above it. For this we use the tower map $g$ in (4.10) and appropriate similarities. By declaring each such map to be the identity elsewhere in $\mathcal{C}_{0}$, we get a map

$$
f_{1}: \mathbb{B}^{n} \rightarrow \mathcal{C}_{1}
$$

where $\mathcal{C}_{1}$ is $\mathcal{C}_{0}$ plus all the new towers placed above each $\Delta_{i}$. The map $f_{1}$ is simply $f_{0}$ followed by all those little tower maps. Because $f_{0}$ was a similarity on $f_{0}^{-1}\left(U_{i}\right)$, and because the bases $\Delta_{i}$ are located in $U_{i}$, the dilatation of $f_{1}$ only depends on $n$ and $\alpha$. In other words, we did not increase the dilatation by this composition because the only nontrivial contribution came from where $f_{0}$ was conformal.

Now we continue in a similar fashion. The walls of all the little towers in $\mathcal{C}_{1}$ are flattened near the points where the children (the grandchildren of $T_{0}$ ) leave $\mathcal{C}_{1}$, and $f_{1}$ is modified so as to become a similarity 
near those points. This modification increases the dilatation but there is no accumulation because the increase only occurs at places where $f_{0}$ was conformal. Then we blow up new (possibly leaning) towers from those newly created similarity neighborhoods. Thus the dilatation of the map $f_{2}: \mathbb{B}^{n} \rightarrow \mathcal{C}_{2}$ will not grow, where, naturally, $f_{2}$ is $f_{1}$ followed by the new even littler tower maps, declared to be the identity outside the bases, and $\mathcal{C}_{2}$ is the union of $\mathcal{C}_{1}$ and the new towers.

The final map $f$ is the limit of the maps $f_{0}, f_{1}, f_{2}, \ldots$ constructed in this manner. Its dilatation in $\mathbb{B}^{n}$ only depends on $n$ and $\alpha$, and it maps $\overline{\mathbb{B}}^{n}$ onto $\bar{D}$, where $D$ is the interior of the union $\mathcal{C}_{0} \cup \mathcal{C}_{1} \cup \mathcal{C}_{2} \cup \ldots$. If the tree is properly arranged, $f$ will be a homeomorphism of the closed unit ball onto $\bar{D}$. It is also clear by construction that the set $F_{T}$ lies on the boundary of $D$, and that we can always arrange the boundary $\partial D$ minus, possibly, the set $F_{T}$, to be of finite Hausdorff $\mathcal{H}_{n-1}$-measure.

This completes the proof of Theorem 4.3.

\section{Proof of the Wall Conjecture in dimension $n=3$.}

Soon after Jussi Väisälä heard about the Wall Conjecture, he devised a simple argument in dimension $n=3$ which also proves the following more general theorem.

Theorem 5.1. (Väisälä, [V6]) Suppose that $G$ is an open set in $\mathbb{R}^{n}$, $n \geq 3$, such that $\check{H}^{1}\left(\mathbb{R}^{n} \cup\{\infty\} \backslash G\right)=0$ and that $\mathbb{R}^{n} \backslash G$ satisfies the condition $c-\mathrm{LLC}_{2}$. Then

$$
\mathcal{H}_{2}(B(x, 2 \operatorname{dist}(x, \partial G)) \cap \partial G) \geq \frac{\pi}{16 c}(\operatorname{dist}(x, \partial G))^{2}
$$

for each $x \in G$.

In the theorem, $\check{H}^{1}$ denotes the first Čech cohomology group with integer coefficients. The $c-\mathrm{LLC}_{2}$ condition means that for every $x$ in $\mathbb{R}^{n} \backslash G$ and $r>0$ points in $\left(\mathbb{R}^{n} \backslash G\right) \backslash \bar{B}(x, r)$ can be joined in $\left(\mathbb{R}^{n} \backslash\right.$ $G) \backslash \bar{B}(x, r / c)$, where $c \geq 1$ is a constant independent of $x$ and $r$.

If $D$ is a $(K-)$ quasiconformal Jordan ball in $\mathbb{R}^{3}$, then $\check{H}^{1}\left(\mathbb{R}^{3} \cup\right.$ $\{\infty\} \backslash D)=0$ by Alexander duality, and $\mathbb{R}^{3} \backslash D$ is $c$-LLC 2 for some $c \geq 1$ depending only on $K$ by a theorem of Gehring and Väisälä [GV]. The letters LLC stand for linear local connectivity. It is also true, and proved by Gehring and Väisälä $[\mathrm{GV}]$, that $\mathbb{R}^{3} \backslash D$ satisfies the following 
$c$ - LLC $_{1}$ condition, which is dual to $c$-LLC 2 : for every $x \in \mathbb{R}^{n} \backslash G$ and $r>0$ points in $\left(\mathbb{R}^{n} \backslash G\right) \cap B(x, r)$ can be joined in $\left(\mathbb{R}^{n} \backslash G\right) \cap B(x, c r)$.

Therefore, Theorem 1.5 follows from Väisälä's Theorem 5.1.

I shall next sketch another proof for Theorem 1.5, but the reader should bear in mind that it is not as elegant as Väisälä's argument and it will not generalize so as to cover Theorem 5.1. But even this proof as such has nothing to do with quasiconformal maps; we shall only employ the LLC condition for the complement. In Problem 5 below in Section 6 we formulate a general conjecture along the lines "quantitative topological conditions imply mass bounds". This type of results have recently been popular in Riemannian geometry; see [GP].

Proof of TheOREM 1.5. We can normalize the situation so that $x=0$ and $\operatorname{dist}(x, \partial D)=1$. It is an easy exercise to check that it is enough to find constants $C_{1}=C_{1}(K) \geq 2$ and $C_{2}=C_{2}(K)>0$ such that

$$
\mathcal{H}_{2}\left(B\left(0, C_{1}\right) \cap \partial D\right) \geq C_{2} .
$$

Next we invoke a lemma which is due to Gehring [G1, Lemma 1]. In the lemma, we denote by $K_{1}$ the decomposition of $\mathbb{R}^{3}$ into closed cubes with vertices in $\mathbb{Z}^{3}$; then write $K_{s}=s K_{1}$ for $s>0$, and denote by $K_{s}^{1}$ the 1 -skeleton of $K_{s}$.

Lemma 5.3. Suppose that a compact set $A$ in $\mathbb{R}^{3}$ satisfies

$$
\mathcal{H}_{2}(A)<\frac{s^{2}}{64}<1
$$

for some $s>0$. Then some translate $A-y=\{a-y: a \in A\}, y \in \mathbb{R}^{3}$, does not meet the 1-skeleton $K_{s}^{1}$.

Now choose the constant $C_{1}>2$ in (5.2) very large and $s>0$ very small (both depending on the constant $c$ in the linear local connectivity condition, hence on $K$ only) and assume that (5.4) holds for $A=\partial D \cap$ $\bar{B}\left(0, C_{1}\right)$. Then the part of the (translated) 1 -skeleton $K_{s}^{1}$ that lies in $B\left(0, C_{1}\right)$ does not meet $\mathbb{R}^{3} \backslash D$, because it does not meet $\partial D$, it is connected, and it meets $D$ near the point 0 . This will lead to a contradiction as follows. One first selects a curve $\gamma_{1}$ in $\mathbb{R}^{3} \backslash D$ that joins some point $w$ on $\partial D$ with $|w|=1$ to a point in $\partial B\left(0, C_{1}\right)$. Then, by using the $\mathrm{LLC}_{2}$ condition, one selects another curve $\gamma_{2}$ joining the 
same points in $\mathbb{R}^{3} \backslash D$, but in such a way that the union $\gamma=\gamma_{1} \cup \gamma_{2}$ will link one of the polygonal circles forming the (translated) 1-skeleton $K_{s}^{1}$. Although at the first glance it seems clear that such a curve $\gamma_{2}$ exists, the selection is not totally trivial; it can be done however.

This linking contradicts the fact that any circle in $D$ is contractible in the complement of $\gamma$, because $\gamma$ lies in $\mathbb{R}^{3} \backslash D$. The theorem follows.

\section{Open problems.}

Problem 1. Prove Theorem 1.1 in all dimensions $n \geq 3$. This can be accomplished if the next question admits a positive answer. Similarly, in that case one can replace double cone points with inner cone points.

Problem 2. Suppose that $F$ is a compact set in $\mathbb{R}^{n}, n \geq 2$, and that $\varphi: F \rightarrow \varphi(F)$ is a quasisymmetric embedding of $F$ into $\mathbb{R}^{n}$. Is it true that the $n$-measure of $\varphi(F)$ is zero if the $n$-measure of $F$ is zero?

The proof in $[\mathrm{H}]$ of Theorem 1.1 would not only work in all dimensions $n \geq 3$ but it would also tremendously simplify, should the answer to this question be yes. In particular, no Sullivan theory of Lipschitz approximations is needed. Note that the answer to the question is no if $n=1$.

Quasisymmetric maps are defined in (3.13), and their basic theory can be found in [TV1], [V2].

Problem 3. What version, if any, of Theorem 1.2 remains true if we only assume that $\partial D$ be of finite $\mathcal{H}_{2}$-measure? By using the tree construction amended by a certain bubble blowing procedure, it is not hard to construct a quasiconformal Jordan domain $D$ in $\mathbb{R}^{n}, n \geq 3$, such that the boundary $\partial D$ has a purely unrectifiable piece of positive $\mathcal{H}_{n-1^{-}}$ measure that transforms onto a set of Hausdorff dimension zero under a quasiconformal map $f: D \rightarrow \mathbb{B}^{n}$, and that the complement $\mathbb{R}^{n} \backslash D$ has no points of $n$-density on $\partial D$. However, I have only been able to construct $D$ in such a way that its boundary has $\sigma$-finite Hausdorff $\mathcal{H}_{n-1}$-measure.

Problem 4. Let $f$ be a quasiconformal map of $\mathbb{B}^{n}$ onto a Jordan domain $D$ in $\mathbb{R}^{n}, n \geq 3$, and suppose that the boundary of $D$ has finite $\mathcal{H}_{n-1}$-measure. When is $f^{-1}: \partial D \rightarrow \partial \mathbb{B}^{n}$ absolutely continuous? The 
best known result to the author is the case when $\partial D$ is $(n-1)$-regular as defined in (3.12). Then $f$ is not only absolutely continuous, but it induces a measure that is $A_{\infty}$ related to $\mathcal{H}_{n-1}$. This result is essentially due to Gehring. (See $[\mathrm{S}, 3.4]$ or $[\mathrm{H}, 2.7]$ ). From the point of view of boundary behavior, regularity is a strong assumption. It does not cover, for instance, maps that can be extended to global quasiconformal maps of $\mathbb{R}^{n}$.

Problem 5. Prove the Wall Conjecture in all dimensions. Related to this, Jussi Väisälä has proposed the following generalized Wall Conjecture, abbreviated $\mathrm{WC}(n, p)$, for all integers $n \geq 2$ and $1 \leq p \leq n-2$. Suppose that $G$ is a homologically trivial open set in $\mathbb{R}^{n}, n \geq 2$, and suppose that $\mathbb{R}^{n} \cup\{\infty\} \backslash G$ is inner $(k, c)$-joinable for all $0 \leq k \leq p-1$. Then the conjecture $\mathrm{WC}(n, p)$ states that

$$
\mathcal{H}_{p+1}(B(x, 2 \operatorname{dist}(x, \partial G)) \cap \partial G) \geq C(c, n) \operatorname{dist}(x, \partial G)^{p+1}
$$

for $x \in G$. The notion of inner joinability was introduced by Väisälä in [V5], where we refer the reader for a precise definition. It suffices to say here that the inner $(0, c)$-joinability is precisely the $c-\mathrm{LLC}_{2}$ condition. Thus Väisälä's Theorem 5.1 implies that $\mathrm{WC}(n, 1)$ is true. It is also not hard to see that $\mathrm{WC}(n, 0)$ is true; note that in this case the second requirement about joinability becomes empty, while the first requirement about $G$ being homologically trivial implies the connectivity of the complement of $G$.

All other cases of $\mathrm{W} C(n, p)$ are open. The wall conjecture as stated in (1.8) would follow from $\mathrm{WC}(n, n-2)$, because Väisälä has shown in [V5] that the complement of a quasiconformal ball is $(k, c)$-joinable for all $0 \leq k \leq n-3$.

Acknowledgements. I wish to thank Fred Gehring, Pekka Koskela, Pertti Mattila, Steffen Rohde, Stephen Semmes, and Jussi Väisälä for helpful and inspiring discussions on the topics related to this paper. I also thank the referee for a careful reading of the manuscript and useful comments.

Added in Proof. After this paper was submitted, two relevant developments took place. First, Semmes (Semmes, Quasisymmetry, measure and a question of Heinonen, this issue) solved Problem 2 above; 
its consequences are discussed in (Heinonen, A Theorem of Semmes and boundary absolute continuity in all dimensions, this issue). In particular, Problem 1 is now solved as well. Second, Väisälä (The Wall Conjecture on Domains in Euclidean Spaces, Preprint, University of Helsinki, 1996) solved the generalized Wall Conjecture as in Problem 5 above. As a joint consequence of the results of Semmes and Väisälä, Theorem 1.2 is true in all dimensions $n \geq 3$, verifying the conjecture made on page 6 before Theorem 1.5. Namely, assuming the Wall Conjecture, the case $n \neq 4$ is already proved in the present paper, and the case $n=4$ can be handled by the aid of the aforementioned result of Semmes as in (Heinonen, this issue).

\section{References.}

[BM] Baernstein, A., Manfredi, J. J., Topics in quasiconformal mappings, Topics in modern harmonic analysis. Proc. Seminar held in Torino and Milano II (1982), 849-862.

[BA] Beurling, A., Ahlfors, L. V., The boundary correspondence under quasiconformal mappings. Acta Math. 96 (1956), 125-142.

[F] Federer, H., Geometric Measure Theory. Springer, 1969.

[FHM] Fernández, J. L., Heinonen, J., Martio, O., Quasilines and conformal mappings. J. Analyse Math. 52 (1989), 117-132.

[G1] Gehring, F. W., The Hausdorff measure of sets which link in Euclidean space. Contributions to Analysis: A Collection of Papers Dedicated to Lipman Bers. Academic Press, 1974.

[G2] Gehring, F. W., Lower dimensional absolute continuity properties of quasiconformal mappings. Math. Proc. Camb. Phil. Soc. 78 (1975), 81-93.

[GP] Greene, R. E., Petersen V. P., Little topology, big volume. Duke Math. J. 67 (1992), 273-290.

[GV] Gehring, F. W., Väisälä , J., The coefficients of quasiconformality of domains in space. Acta Math. 114 (1965), 1-70.

[Ha] Hanson, B., Quasiconformal analogues of a theorem of Smirnov. Math. Scand. 75 (1994), 133-149.

[H] Heinonen, J., The boundary absolute continuity of quasiconformal mappings. Amer. J. Math. 116 (1994), 1545-1567.

[HK] Heinonen, J., Koskela, P., The boundary distortion of a quasiconformal mapping. Pacific J. Math. 165 (1994), 93-114. 
[KR] Koskela, P., Rohde, S., Hausdorff dimension and mean porosity. To appear in Math. Ann.

[MM] Malý, J., Martio, O., Lusin's condition (N) and mappings of the class $W^{1, n}$. J. reine angew. Math. 458 (1995), 19-36.

[M] Mattila, P., Geometry of Sets and Measures in Euclidean Spaces. Cambridge Studies in Advanced Mathematics 44. Cambridge University Press, 1995.

[Re] Reshetnyak, Yu. G., Some geometric properties of functions and mappings with generalized derivatives (Russian). $\begin{array}{lllll}\text { Sibirsk. } & \text { Mat. } & \text { Zh. } & \mathbf{7}\end{array}$ (1966), 886-919.

[R] Riesz, F., Riesz, M., Über Randwerte einer analytischen Funktionen. Quatrième Congrès des Math. Scand. Stockholm (1916), 27-44.

[S] Semmes, S., Bilipschitz mappings and strong $A_{\infty}$-weights. Ann. Acad. Sci. Fenn. Series A I Math. 18 (1993), 211-248.

[TV1] Tukia, P., Väisälä, J., Quasisymmetric embeddings of metric spaces. Ann. Acad. Sci. Fenn. Ser. A I Math. 5 (1980), 97-114.

[TV2] Tukia, P., Väisälä, J., Lipschitz and quasiconformal approximation and extension. Ann. Acad. Sci. Fenn. Ser. A I Math. 6 (1981), 303-342.

[V1] Väisälä, J., Lectures on n-dimensional quasiconformal mappings. Lecture Notes in Math. 229, Springer-Verlag, 1971.

[V2] Väisälä, J., Quasi-symmetric embeddings in Euclidean spaces. Trans. Amer. Math. Soc. 264 (1981), 191-204.

[V3] Väisälä, J., Quasimöbius maps. J. Analyse Math. 44 (1984/85), 218234.

[V4] Väisälä, J., Quasiconformal maps and positive boundary measure. Analysis 9 (1989), 205-216.

[V5] Väisälä, J., Metric duality in Euclidean spaces. To appear in Math. Scand.

[V6] Väisälä, J., Unpublished notes. 1995.

Recibido: 25 de agosto de 1.995

Juha Heinonen*

University of Michigan

Department of Mathematics

Ann Arbor, MI 48109, U.S.A. juha@math.1sa.umich.edu

\footnotetext{
* Supported in part by NSF and the Academy of Finland. The author is a Sloan fellow.
} 